\title{
La importancia de los gobernantes invisibles en la democracia. Un estudio de la «estructura de poder latente» de dos Gobiernos en España (2004 y 2012)
}

\author{
The importance of invisible rulers in democracy. A study of the "latent \\ power structure" of two Executives in Spain (2004 and 2012)
}

\author{
ANDRÉS VILLENA-OLIVER \\ Universidad de Málaga
}

\section{BERNABÉ ALDEGUER-CERDÁ}

Universidad de Alicante

\section{Cómo citar/Citation}

Villena-Oliver, A. y Aldeguer-Cerdá, B. (2017). La importancia de los gobernantes invisibles en la democracia. Un estudio de la "estructura de poder latente» de dos Gobiernos en España (2004 y 2012). Revista Española de Ciencia Política, 45 67-94. Doi: https://doi.org/10.21308/recp.45.03

\section{Resumen}

Este artículo analiza la composición de dos Gobiernos españoles desde la perspectiva de las redes de relaciones existentes entre sus componentes, de manera previa a la formación del Ejecutivo. Dicha perspectiva nos informa de cómo determinados actores individuales y grupos de poder influyen potencialmente en la formación de un Gobierno y en su acción política. Para realizar este estudio, se define la estructura de poder latente (EPL) como un conjunto de expresidentes, exministros y directivos empresariales que mantienen relaciones significativas con los miembros del Ejecutivo a partir de contactos establecidos en el pasado. La consideración de esta estructura permite concebir los Gobiernos como agencias públicas dotadas de una mayor concentración de relaciones de lo que se puede apreciar a partir de sus componentes plasmados en la estructura formal. En este sentido, la EPL muestra, por una parte, que los Ejecutivos mantienen vínculos con numerosos grupos, especialmente con grandes empresas privadas; y por otra, que estos Gobiernos son, en realidad, una red de miembros dotada de una mayor cohesión interna que la identificable de forma patente a partir de los altos cargos oficialmente reclutados.

Palabras clave: cohesión, élites, estructura de poder latente, Gobierno, redes sociales. 


\begin{abstract}
This article aims to analyze the composition of two recent Spanish governments from the point of view of the relations between their members before the executives were formed. This perspective lets us know how certain individual actors and power groups are potentially able to influence the government formation process and its political action. In order to carry out this study, the concept of the 'Latent Power Structure' is defined as a set of former presidents, ex ministers and enterprise directors or managers that have established relevant social ties with the members of a government due to having coincided in certain institutions in the past. Taking the Latent Power Structure into account allows us to conceive governments as public agencies that accumulate a higher degree of social relations than it can be inferred out of their formal structure. In this sense, the Latent Power Structure shows, on the one hand, that the executives have relevant social ties with power groups - especially big private firms-; and, on the other hand, these governments are, in fact, networks of members endowed with a greater internal cohesion than it is clearly identifiable from the high ranking officials formally recruited.
\end{abstract}

Keywords: cohesion, elites, latent power structure, Government, social networks.

\title{
INTRODUCCIÓN: GOBIERNOS OFICIALES E INFLUENCIA POTENCIAL
}

Este trabajo estudia, desde la perspectiva del análisis de redes sociales, los fundamentos de la configuración del poder en dos Gobiernos, con la aportación de un enfoque empírico escasamente empleado. Los estudios sobre el poder se han limitado, a menudo, a la observación y al estudio de un conjunto de posiciones oficiales y evidentes por medio de un análisis institucionalista o posicional. En este sentido, Putnam considera que las principales instituciones estatales proporcionan un mapa útil para el diagnóstico de las relaciones de poder y, por lo tanto, que los dirigentes situados en los puestos más elevados de dichas instituciones, desde una perspectiva jerárquico-vertical, son los políticamente poderosos (Putnam, 1976). Sin embargo, esta consideración no concede suficiente importancia al hecho de que estos miembros del poder ejecutivo se encuentran, en realidad, vinculados a otros individuos que, aun sin pertenecer a la estructura oficial del Gobierno, ejercen una importancia potencial sobre los miembros gubernamentales, tal y como Bobbio puso de manifiesto al desarrollar la noción «the invisible Government», la cual reside en el seno del Estado democrático y no se manifiesta en los procedimientos democráticos de rendición de cuentas (Bobbio, 2013). En La red del poder, Cervera destaca la brecha o distancia entre dos poderes paralelos, el poder ejecutado u oficial, y el poder real o decisorio, siendo el primero el visible o institucional, y el segundo, el compuesto por los superiores desconocidos, oculto, que no se manifiesta explícitamente (Cervera, 1984).

En la medida en que este poder de carácter latente puede vulnerar la realización de la responsabilidad democrática propia del sistema representativo ejercida sobre el cargo público en tanto que agente (Sánchez de Dios, 2006), la razón principal por la 
cual se aborda la presente propuesta de estudio de las relaciones de influencia subyacente radica en el propósito de identificar, definir y caracterizar a los conjuntos humanos compuestos por ex altos funcionarios gubernamentales que vendrían a ocupar posiciones relevantes de mediación entre el Gobierno y las instituciones civiles, sociales o empresariales de las que forman parte: grandes empresas, Gobiernos regionales o grandes instituciones internacionales.

La influencia potencial de estos individuos se basa en dos factores fundamentales:

1. En primer lugar, en el hecho de haber ejercido funciones institucionales formales en: a) Ejecutivos previos, así como en otros ámbitos tales como el propio Parlamento nacional, teniendo en cuenta la importancia del componente institucional en la definición de las carreras políticas (Borchert, 2003), y b) empresas privadas, partidos políticos, además de otras instituciones.

2. En segundo lugar, estas personas están actualmente trabajando para otros grupos de poder (lobbies, corporaciones empresariales, instituciones financieras, etc.), lo cual implica una conexión potencial entre el entramado gubernamental y los grupos de interés a los que estos pertenecen. Aunque puede haber variaciones en función de la ideología del Gobierno, la consideración de estas influencias, que podemos denominar latentes, revela un Ejecutivo dotado de una estructura relacional con más recursos de poder, así como de un mayor grado de cohesión y de unidad ${ }^{1}$.

Esta perspectiva nos ofrece una explicación alternativa a la de las denominadas "carreras postministeriales», en tanto que idea asiduamente relacionada con la "puerta giratoria» o revolving door. En este sentido, Musella (2015), al estudiar la trayectoria individual de un conjunto de expresidentes, ha afirmado que es durante su experiencia de Gobierno cuando las posibilidades de futuras colaboraciones con empresas y otras instituciones aparecen, lo que incluso puede llegar a afectar a las propias políticas públicas. Aunque un gran número de investigaciones se han dedicado al estudio de los movimientos y de las pautas de circulación de los exministros hacia las instituciones públicas y privadas después de haber dejado el cargo (Rose, 1987; Thiébault, 1991; Suleiman y Mendras, 1995; Parrado, 1996; Rodríguez Teruel, 2010; Botella et al., 2011), se ha prestado menos atención a la influencia que estos ex altos cargos pueden llegar a ejercer sobre los Gobiernos del mismo signo político a los que pertenecieron $\mathrm{y}$, en consecuencia, a cómo ciertos Ejecutivos quedan potencialmente conectados con los grupos de poder que han reclutado a estos exministros (y a cómo, en suma, estos nuevos grupos, tales como empresas e instituciones supranacionales, pueden adquirir contactos privilegiados con los organismos gubernamentales). De esta manera, el

1. Luengo (2016) formulará el concepto de «sociedad conyugal» para poner de manifiesto la importancia de las redes definidas por relaciones de parentesco en la constitución de las élites liberales del siglo xix en Espańa. 
estudio de la estructura de poder latente revela la posibilidad conceptual y analítica de conocer el importante conjunto de conexiones potenciales que un determinado Gobierno entabla con numerosos grupos e instituciones de poder, más allá de un análisis posicional o institucional y formal.

Según nuestra propuesta, esta investigación centra sus propósitos en los siguientes objetivos:

1. En primer lugar, en la medida en que se trata de comprender el papel potencial de determinados influyentes externos en los Ejecutivos, se evalúa el grado de influencia relacional de un conjunto de exministros y de otros dirigentes que, aun no habiendo sido reclutados por un determinado Gobierno, están, sin embargo, vinculados a algunos líderes de dicho Ejecutivo. Estos componen lo que denominamos la estructura de poder latente (EPL).

2. En segundo lugar, para medir esta influencia latente, estudiamos cómo algunas características importantes de los Gobiernos, tales como su cohesión interna o la cantidad de vínculos establecidos con grupos de poder externos, se ven modificadas cuando la mencionada EPL es tenida en cuenta.

3. En tercer lugar, y con el fin de demostrar que la EPL representa un elemento estructural que prevalece sobre los cambios electorales, analizamos cómo los Gobiernos formados por los distintos partidos políticos se ven afectados por sus respectivas estructuras latentes.

4. En cuarto lugar, y una vez que se conozca con más detalle la influencia de las EPL sobre diferentes Gobiernos, se estudia su papel y su relevancia en el proceso de renovación gubernamental en el marco del bipartidismo propio de la democracia española.

El artículo se estructura en tres partes claramente diferenciadas. Se presenta, en primer lugar, el marco teórico relativo a los procesos de reproducción social y las estructuras del poder latente en virtud de las lógicas de funcionamiento del sistema político democrático. En un segundo apartado se presenta el marco analítico y la metodología del trabajo, mediante la construcción de la EPL, presentándose las hipótesis de trabajo, las fuentes de datos y la operacionalización conceptual requerida para examinar las diferencias y las relaciones entre las redes oficiales y las redes de Gobierno ampliado. Así pues, el análisis de los datos abarca tanto la estructura de la red social conformada por cada uno de los Gobiernos a partir de los contactos establecidos entre estos en la constitución del Ejecutivo como los componentes de la denominada EPL, añadiendo a las redes oficiales gubernamentales las de Gobierno ampliado. Finalmente, se presentan los resultados del análisis de redes sociales (ARS), por medio de cuatro epígrafes. Así, en primer lugar se analiza qué características de los Gobiernos se ven modificadas cuando las EPL son consideradas. En segundo lugar, se estudia si se puede deducir un determinado patrón de circulación o de renovación de las élites vinculado a la idea de la «cadena de oportunidades». En tercer lugar, se comprueba si el grado de cohesión interna gubernamental se incrementa significativamente cuando la EPL es 
introducida. En cuarto y último lugar, se observa si la posición relacional de algunos de los miembros del Gobierno se ve o no notablemente modificada cuando se considera la EPL.

\section{MARCO TEÓRICO: LA LÓGICA DEL GOBIERNO DEMOCRÁTICO, LOS PROCESOS DE REPRODUCCIÓN SOCIAL Y LAS ESTRUCTURAS DE PODER LATENTE}

Mayoritariamente opuestas al diagnóstico social marxista, las teorías sobre las élites han reflexionado sobre la manera en la que la administración y la distribución del poder tienen lugar en las sociedades modernas (Bottomore, 1993; Meisel, 1958). En este sentido, los intelectuales maquiavelistas (Burnham, 1943), tales como Mosca (1939), Pareto (1917) y Michels (1915), afirman ${ }^{2}$ que la democracia real se fundamenta en un inevitable ejercicio de dominación por parte de ciertas minorías en el ejercicio del poder, en el marco de la denominada «ley de hierro de la oligarquía». Esta dominación se basa en dos condiciones principales:

1. En primer lugar, en un alto grado de cohesión interna, lo que concede a las minorías dominantes un mayor grado de organización que el de la masa o multitud (Mosca, 1939).

2. En segundo lugar, en el hecho de que los miembros de dichas minorías poseen características extraordinarias, así como recursos sociales que las hacen «superiores» a las personas a las que gobiernan (id.).

Estos recursos o fuentes de poder no son necesariamente cualidades naturales, en la medida en que un individuo pueda heredar una gran suma de dinero o estar fuertemente conectado a un conjunto de grandes empresas privadas, y están, además, sujetas a cambios a lo largo del tiempo. Este último hecho convierte el ejercicio de la «dominación legal» (Weber, 1922) en un equilibrio dinámico en el que la "clase dominante» se renueva constantemente. Pareto (1917) describe este fenómeno de cambio continuo como la «circulación de las élites»: un proceso de renovación de la clase dominante en el que la incorporación de nuevos elementos refuerza y actualiza la superioridad y el poder de la élite. La plena vigencia de estos conceptos clásicos sobre el poder ha sido demostrada recientemente al afirmarse que las élites tienden a determinar y a cambiar constantemente con el propósito de adaptarse a las alteraciones más recientes acaecidas en el entorno social, tales como la globalización de las finanzas o los problemas derivados del cambio climático (Morgan et al., 2015).

2. En esta sección se cita el año de la versión original del libro, a fin de presentar la discusión sobre el poder en un orden cronológico. 
Ciertos estudios en los Estados Unidos desarrollados tras la Segunda Guerra Mundial permitieron reforzar la convicción de que las minorías gobernantes renovaban de forma permanente sus recursos y fuentes de poder, y que la cohesión y las conexiones con determinados grupos influyentes de la sociedad devenían en los factores más relevantes para explicar dicha mutación constante. En este sentido, Mills (1956) definió la relevancia de la élite americana como resultado de la confluencia de tres tipos diferentes de «círculos superiores»: el político, el de los negocios y el de los comités militares, todos los cuales definirían una red en la cual son adoptadas las decisiones nacionales más relevantes. Mills no solo analiza quiénes son las personas más ricas o poderosas; de hecho, sugiere que la combinación institucional de los tres círculos, por una parte, y la red cohesiva que relaciona a sus líderes de forma individual, por otra, constituyen una condición estructural para entender la configuración del poder en las democracias capitalistas avanzadas. Según Mills, así como también de acuerdo con Domhoff (1969), la circulación de la élite consiste en un conjunto de movimientos que se producen desde una de las instituciones decisivas hacia alguna de las otras. Además, los líderes más jóvenes son reclutados desde las universidades elitistas pertenecientes a la denominada «Ivy League» y desde determinados clubes sociales, de tal manera que todo este proceso de reclutamiento y circulación se produce en torno a una comunidad de la élite dotada de una profunda conciencia de clase (Mills, 1956). Podemos considerar, por tanto, a efectos de análisis y de explicación, la existencia de un cierto proceso de bloqueo y endogamia en los procesos de circulación, por medio de los cuales un subsistema elitista se garantiza un suministro constante de recursos de poder, en la definición del party political career management (De Visser y Van der Meer, 2010).

Las principales hipótesis y conclusiones de Mills fueron impugnadas por el grupo académico de los «pluralistas», cuyos trabajos concluyen que, en lugar de una élite dominante unificada, existen diferentes tipos de liderazgo y de influencia dispersos entre distintos grupos de la sociedad. Estos dirigentes estarían especializados en determinados ámbitos económicos o políticos y, por lo tanto, se limitarían a participar y a involucrarse políticamente en una estrecha gama de cuestiones. Estos estudios, inicialmente desarrollados, entre otros, por Dahl (1960), Polsby (1980) y Laumann y Pappi (1976), se centraron en la estructura de ciertas ciudades estadounidenses -como New Haven en el caso de la investigación de Dahl-, pero no así en las élites nacionales, como sí abordaron tanto Mills como Domhoff o Miliband (1969). Los estudios pluralistas ponen de manifiesto la existencia de grupos políticos sobre los que no opera una significativa influencia procedente del mundo de los negocios, y cuya motivación vendría dada por un interés centrado en una serie de temas específicos. Las investigaciones de tal espectro doctrinal destacarían, igualmente, el papel clave de los políticos, que tenderían a coordinar y agregar las demandas de los diferentes grupos sociales y económicos, con el propósito de maximizar los resultados del sistema para beneficiar a la ciudadanía. De esta manera, conforme a la formulación realizada por Bachrach y Baratz en Two faces of power, todo proceso de adopción de decisiones se desarrolla entre una dimensión institucional y otra en la que se producen conflictos no visibles en la faz pública del ejercicio del poder (Bachrach y Baratz, 1962). 
Los estudios pluralistas de origen norteamericano y su concepción de una multiplicidad de actores que interactúan entre sí de forma más o menos equilibrada con el fin de alcanzar sus objetivos tuvieron una influencia relevante en lo que más recientemente se ha denominado el enfoque de las «redes políticas» (Degenne, y Forsé, 1999; De Nooy et al., 2005; Lusher et al., 2013), dedicado a analizar la adopción de decisiones en las comunidades, así como desde la consideración de las llamadas «comunidades epistémicas», en el caso del estudio y análisis de las políticas públicas (Haas, 1989; 1992; Castells, 2006).

Las redes políticas pueden interpretarse en un sentido amplio como un conjunto de lazos de los actores gubernamentales con otros actores sociales y estatales (Rhodes y Marsh, 1992). Según este enfoque, ligado al paradigma de la "gobernanza» (Canales, 2015; Del Campo, 2014; Kooiman, 2005; Prats, 2005), la pérdida de poder estatal ha conducido a la existencia de un conjunto de relaciones formales e informales entre instituciones públicas y privadas que movilizan recursos dispersos, de manera que la acción colectiva puede ser orquestada hacia la solución política de un problema común (Kenis y Schneider, 1991). En este nuevo marco, las técnicas de análisis de redes sociales se aplican para estudiar las relaciones entre los componentes estructurales de la red política, así como los resultados en los que derivaría el intercambio informal y los vínculos existentes entre los actores (Knocke, 2001). De esta manera, se constata que aquel nodo que ostente la posición de mayor centralidad en el seno de una organización caracterizada por este tipo de redes será el más influyente a la hora de determinar los resultados finales de una determinada decisión o negociación (Laumann et al., 1977; Hoffmann-Lange, 1989).

Los vínculos existentes entre los líderes de diferentes instituciones fueron examinados también por Moore (1979) y Higley et al. (1991). En un intento por hallar un equilibrio entre las hipótesis elitistas y pluralistas, Moore concibió la estructura de poder en los Estados Unidos como una red de vínculos entre los dirigentes de distintas procedencias institucionales. Esta red muestra un núcleo cohesionado conformado por personas que, con perfiles semejantes y estrechas relaciones, habrían participado en las mismas instituciones. Dicho núcleo queda integrado, sobre todo, por políticos y ejecutivos de las grandes corporaciones empresariales que, a su vez, quedan conectados con una serie de grupos de poder de elevada especialización y cualificación técnica. Esta estructura compatibiliza la existencia de un núcleo altamente cohesionado con una importante cantidad de conexiones con grupos de poder parcial, como una forma de autogobierno reticular Higley et al. (1991). Esta conclusión debe ser contrastada con la que se extrae del estudio realizado por Heinz et al. (1993), en el cual se analizan las relaciones entre los representantes de los distintos grupos de interés y los dirigentes de las agencias federales y del Parlamento estadounidense. En este marco, el Gobierno federal se concibe como una esfera cuyo centro está vacío, en la medida en que no se detecta ningún tipo de élite que sea capaz de mediar entre los intereses contrapuestos o yuxtapuestos, hecho que constata la ausencia de un centro cohesionado y que se atribuye a la debilidad del Estado.

A partir de los anteriores estudios, puede concebirse un Gobierno como una agencia pública inserta dentro una compleja estructura de poder. Si consideramos que el 
proceso de constitución de un Ejecutivo se lleva a cabo en un entorno de grupos de poder que ejerce una influencia significativa sobre dicho Gobierno, podremos comprender mejor las principales decisiones y características del mismo. Los procesos de formación gubernamental y sus dinámicas en el marco de un sistema político parlamentario se sitúan en el corazón mismo de la democracia representativa (Laver y Shepsle, 1996) y un análisis exhaustivo de una estructura gubernamental puede proporcionar información relevante acerca del entorno social en el que el poder ejecutivo se ha constituido.

Aunque la EPL es un concepto original, se refuerza y adquiere consistencia teórica a partir de reflexiones preexistentes acerca de las élites de poder. Por ejemplo, Mills situó lo que denominaba como el «directorio político», rodeado por los círculos empresariales y militares (Mills, 1956). Para dicho autor, un análisis de la élite tendría que considerar la existencia de esas conexiones. Por su parte, Pareto (1917) distinguía entre una élite "de gobierno» y una élite de «no gobierno», afirmando que si bien ambas eran poderosas e influyentes, solo una gobernaba de manera oficial por medio del entramado institucional. Otros autores han subrayado la influencia de los expresidentes gubernamentales para determinar la elección de un determinado candidato presidencial (Gil Mendieta y Schmidt, 2005). El historiador Tunón de Lara (1967) afirmó que, aparte del Gobierno, hay que tener en cuenta la existencia de grupos de poder que ejercen un alto grado de influencia en la sociedad, por lo que sugiere el término «bloque de poder» para referirse a la estructura de los centros de adopción de decisiones en la que el Gobierno de la nación se encuentra inserto. En el sentido expresado por Bottomore, la estructura formal de gobierno quedaría sometida a grupos e individuos que vendrían a ejercer su poder desde fuera del sistema formal (Bottomore, 1993). Más recientemente, Van Apeldoorn y De Graaff (2014) han estudiado la relación entre determinados grupos de influencia y los funcionarios de más alto rango especializados en la política exterior gubernamental estadounidense desde la presidencia de Bill Clinton hasta la de Barack Obama. Estos autores exponen el vínculo existente entre las grandes corporaciones y una serie de "policy-planning networks» o redes de planificación política con las principales decisiones de política exterior adoptadas por los Estados Unidos.

\section{CONSTRUCCIÓN DE LA ESTRUCTURA DE PODER LATENTE: MARCO ANALÍTICO Y METODOLOGÍA}

En el presente apartado se delinean los aspectos analíticos y metodológicos que han vehiculado el acopio de la evidencia empírica y el análisis de la misma.

A la luz de los antecedentes de carácter teórico y conceptual expuestos, y considerando la formulación de propósitos realizada, las hipótesis sobre las que se va a sustentar el diseño metodológico y la comprobación empírica de este trabajo son las siguientes: 
1. Hipótesis de la EPL. Cuando se tiene en cuenta una serie de miembros especialmente relacionados con los altos cargos del Ejecutivo, la cohesión interna de la estructura de la red del Gobierno resultante aumenta notablemente, definiendo una estructura informal con un alto grado de organización interna, que supera la definición oficial de Gobierno.

2. Hipótesis de los recursos relacionales latentes. Algunos de los miembros oficiales del Gobierno ven incrementada su centralidad en el gobierno ampliado. Por lo tanto, la red que resulta de la consideración de la EPL revela la existencia de recursos relacionales que no habrían sido advertidos de no haber sido considerados estos sujetos. La estructura de la red social gubernamental se ve modificada, con individuos que adquieren una centralidad superior a las de otros.

3. Hipótesis de la "cadena de oportunidades». Cuando se constituye un Gobierno, algunos de los ex altos cargos que no han vuelto a ser reclutados dejan vacantes en el Ejecutivo, las cuales son ocupadas por líderes relacionados con aquellos altos cargos salientes, lo cual mantiene a estos últimos conectados con el Gobierno. Estos movimientos reflejan un proceso que favorece la circulación interna de los miembros de la élite y que puede ser considerado como un mecanismo de renovación endogámica o cerrada.

En cuanto a los casos objeto de estudio y como se ha señalado más arriba, el análisis empírico se centra en la estructura relacional de dos Gobiernos españoles de formación relativamente reciente: el constituido en el año 2004 por el Partido Socialista Obrero Español (PSOE) y el formado en el 2012 por el Partido Popular (PP). En ambos casos, el partido político que conformó el Ejecutivo lo hizo tras permanecer durante ocho años en la oposición parlamentaria: el PSOE estuvo en la oposición desde 1996 a 2004 y el PP entre el año 2004 y el 2012. De esta forma, se han escogido dos Gobiernos comparables formados bajo diferentes circunstancias políticas y económicas. En ambos casos, además, converge la circunstancia de que, en el marco del bipartidismo imperfecto presente en la fase de consolidación democrática, ambos partidos mantienen vínculos con un conjunto de ministros y de altos cargos procedentes de Ejecutivos previos -para el PSOE, los altos cargos de Gobiernos presididos por Felipe González; para el PP, los de los presididos por José María Aznar-; esta circunstancia los hace asimilables, a diferencia, por ejemplo, de los Gobiernos formados por el PSOE en el 2008 y por el PP en el 2016, cuyos miembros ya han tenido cuatro años para fortalecer sus lazos relacionales entre sí.

Para el estudio de los conjuntos humanos que componen estos Gobiernos, se adopta una definición ampliada de Gobierno en la que a los ministros -que constituyen el Ejecutivo desde una concepción jurídica- se añaden otros altos cargos de menor rango como los secretarios de Estado y los subsecretarios, en línea con las consideraciones realizadas por Baena en su estudio sobre la Administración pública española (1993), y de acuerdo con la formulación que de los órganos de colaboración cualificados del Gobierno, en la arena de engarce político-administrativa, realiza la Ley 50/1997, de 27 de noviembre, del Gobierno. De esta forma, los cargos objeto de análisis son los siguientes: el presidente del Gobierno y del Consejo de Ministros; los vicepresidentes; los ministros; los 
secretarios de Estado, y los subsecretarios. Todo ello constituye una población (N) de 69 personas para el Gobierno del PSOE en el 2004 y de 70 para el PP en el 2012.

\section{Las redes sociales de los Gobiernos}

Para la consecución de los propósitos más arriba expuestos, se efectúa un ARS de la estructura reticular determinada por los miembros escogidos para cada Gobierno (presidente, ministros, secretarios de Estado y subsecretarios). Se debe insistir en que lo que se analiza son las relaciones entre dichos miembros gubernamentales de manera previa a la constitución de cada uno de los Ejecutivos, con el objeto de poner de manifiesto los lazos sociales que existen entre los componentes de la mencionada agencia pública en el momento del inicio de cada una de las legislaturas y, con ello, estudiar la estructura reticular existente al comienzo del período. Estas relaciones previas se producen en diversos ámbitos. Para el presente estudio, y con la finalidad de operacionalizar los vínculos existentes acudiendo únicamente a fuentes de datos oficiales y objetivas, se presta una especial atención a cinco dimensiones institucionales distintas:

1. Ejecutivos anteriores del mismo signo político al del gobierno en el que se integran los individuos seleccionados.

2. Grupos parlamentarios, en particular, los del PP y PSOE en el Congreso de los Diputados.

3. Estructura directiva de cada uno de los dos partidos, PP y PSOE: en el caso del PSOE, la Comisión Ejecutiva Federal; en el del PP, el Comité Ejecutivo.

4. Órganos directivos de empresas privadas.

5. Burócratas de élite, o miembros de los denominados altos cuerpos funcionariales según la clasificación efectuada por Baena del Alcázar (1999), entre otros: abogados del Estado, administradores civiles del Estado, técnicos comerciales y economistas del Estado, inspectores de Hacienda, catedráticos de Universidad o componentes de la carrera diplomática.

Estas cinco dimensiones fueron escogidas para abarcar las instituciones formales más relevantes en que se vertebra la arquitectura institucional de toda sociedad democrática, de acuerdo con los enfoques tradicionales sobre las élites en España (Baena y Pizarro, 1985), considerando el área de conexión entre el ámbito político y el ámbito administrativo ${ }^{3}$.

3. El diseño de la investigación no ha obviado la importancia de dimensiones relacionales tales como, por ejemplo, las redes y los vínculos informales establecidos entre los miembros de cada Ejecutivo. La consideración de esta dimensión hubiera exigido la realización de una entrevista a cada uno de los miembros de los dos Gobiernos, por lo que, como se ha dicho más arriba, se ha optado por la consulta de fuentes oficiales. 
Este trabajo estudia las relaciones existentes entre los altos cargos de cada Gobierno que hayan coincidido durante el mismo período en una o más de las dimensiones institucionales antes mencionadas, siempre de manera previa a la formación del Ejecutivo. La intensidad relacional, definida en la teoría de ARS como la fuerza del lazo que conecta a dos actores (Hanneman, 2005), se calcula a partir del número de ańos durante los cuales dichos actores permanecieron en la misma dimensión institucional. De esta forma, se obtiene la representación de una red social a partir de cada una de las cinco dimensiones relacionales estudiadas, lo cual permitirá construir una estructura reticular multidimensional o multiplex en la que integrar las citadas cinco dimensiones institucionales, sumando el valor de los vínculos entre cada par de actores en dichas dimensiones. A modo de ejemplo, si los individuos A y B se relacionaron durante dos años en la dimensión empresarial y durante tres en la dimensión parlamentaria, aparecerán relacionados durante cinco ańos en la red multidimensional; la relación entre A y B tiene una intensidad de 5 , lo que permite considerar el número de años durante los que se mantiene un contacto en diferentes dimensiones de la sociedad; existen alternativas, como la de considerar únicamente en cuántas dimensiones institucionales coinciden los individuos; empleando este último criterio, A y B tendrían una intensidad relacional de 2, pudiendo ser esta como máximo de 5 , al ser 5 las dimensiones empleadas. El resultado de los análisis es similar empleando un criterio y el otro.

Para probar la validez de las hipótesis y lograr la consecución de los objetivos de investigación enumerados, las redes multidimensionales se analizan con el empleo de técnicas de ARS, conforme a la metodología formulada por Wasserman (1994) y por Scott (2000), entre otros. El ARS estudia el comportamiento social de las personas dejando en un segundo plano sus atributos característicos, centrando la atención en el conjunto de los vínculos establecidos entre ellos (Hanneman y Riddle, 2005). Para realizar este tipo de análisis, se define de manera sintética una red social como un conjunto de relaciones establecidas entre una serie de nodos o actores (Kadushin, 2012).

El acopio de la evidencia empírica se ha realizado con base en las siguientes fuentes de carácter público y oficial: Boletín Oficial del Estado; boletines y/o diarios oficiales de las diferentes comunidades autónomas; el Archivo del Congreso de los Diputados, del Senado y de las diferentes Asambleas Legislativas autonómicas; el organigrama de los diferentes ministerios; el currículum vitae publicado en la página web de los ministerios correspondientes; las páginas web oficiales de los partidos políticos que forman parte del estudio (PP y PSOE), así como de sus federaciones (PSOE) o delegaciones regionales (PP); buscadores de empresas y ejecutivos empresariales con acceso al Boletin Oficial del Registro Mercantil, tales como Axesor y Empresia, y otras fuentes complementarias de naturaleza bibliográfica (libros y revistas de carácter divulgativo y académico).

Finalmente, y con el propósito de verificar las informaciones obtenidas a la luz de las fuentes enumeradas, se procedió a su contraste por medio de la oficina del Ministerio de Hacienda y Función Pública competente en la gestión y resolución de las eventuales incompatibilidades profesionales que pudieran afectar a los altos cargos de la Administración del Estado. Para ello, se solicitó a dicho ministerio un informe de posibles incompatibilidades respecto a cada uno de los 69 miembros del Gobierno 
del PSOE en el año 2004 y de cada uno de los 70 componentes del Ejecutivo del PP en el 2012. La totalidad de los informes sirvió para contrastar la información obtenida y para ańadir, en su caso, los vínculos institucionales que no hubiesen podido ser deducidos de las fuentes anteriormente mencionadas.

\section{La construcción de la estructura de poder latente}

Como se ha expuesto más arriba, una vez analizada la estructura de la red social que conforma cada uno de los Gobiernos, se procede a la inserción de la denominada EPL, que se define como una red de relaciones establecida entre los principales miembros componentes de un determinado Gobierno, por una parte, y un conjunto de dirigentes de otros grupos de poder pertenecientes o procedentes de los cinco ámbitos institucionales enumerados en el epígrafe previo (las redes sociales de los gobiernos), con los cuales el Ejecutivo mantiene conexiones debido a que unos y otros coincidieron anteriormente, por otra parte.

Los componentes de una determinada EPL permiten valorar la influencia potencial que determinados grupos de poder adquieren en un momento de vigencia institucional, lo cual convierte a tales grupos de poder en potenciales elementos de influencia sobre el Ejecutivo democrático. A modo de ejemplo, si en el año $2012 \mathrm{el}$ individuo $\mathrm{X}$ es miembro del Gobierno, habiendo trabajado previamente, durante ocho años, como secretario de Estado de un ministro precedente $(Y)$, se considera que $\mathrm{Y}$ es un contacto relevante de $\mathrm{X}$, y que, a pesar de que $\mathrm{Y}$ esté dirigiendo una empresa financiera en el momento presente, este se encuentra en condiciones de ejercer una influencia potencial sobre el comportamiento gubernamental de $\mathrm{X}$ y, por tanto, sobre el Gobierno al que X pertenece. La mayoría de los miembros de la EPL son exministros o expresidentes de Gobierno que, tras haber dejado de ejercer el cargo institucional, han pasado a formar parte de los consejos de administración de determinadas empresas privadas.

Una vez definida la EPL, se presentan los criterios para su correcta operacionalización. A pesar de que los distintos miembros gubernamentales han establecido numerosas relaciones con otros líderes y dirigentes en el pasado, que serían potencialmente influyentes sobre cada uno de los componentes del Ejecutivo, a los efectos del presente trabajo la EPL queda restringida al conjunto de los exministros, portavoces parlamentarios, líderes de los partidos y presidentes de las grandes empresas asociados de manera recurrente y significativa a los miembros del Gobierno. Consideraremos que un actor queda asociado a un miembro de los Gobiernos examinados si este reclutó en el pasado o bien a dos o más miembros de dicho Gobierno, o bien a uno solo pero durante un plazo mínimo de cuatro años - por lo general, el período de un mandato político-. Siguiendo este procedimiento, se obtienen veinte miembros de la EPL por cada Gobierno analizado. La principal razón por la que se restringe la EPL a los cargos arriba expuestos reside en la dificultad para encontrar por la vía de las fuentes oficiales vinculaciones en el seno de otros grupos influyentes, como podrían ser los organismos 
directivos de los sindicatos, determinadas organizaciones de la Iglesia católica, otros grupos influyentes, etc. Se considera que una forma complementaria que optimizaría el resultado de la búsqueda sería la posibilidad de entrevistar a los miembros del Gobierno y preguntarles por las personas más influyentes para cada uno de ellos, lo que ha resultado imposible para la realización del presente estudio.

\section{RESULTADOS. ANÁLISIS DE REDES SOCIALES (ARS)}

En este apartado se exponen los resultados del ARS aplicado a los gobiernos del PSOE y del PP, de acuerdo con lo expuesto en el apartado anterior.

Los gráficos 1, 2, 3 y 4 muestran la estructura relacional de los Gobiernos del PP y del PSOE en el momento de constitución de los Ejecutivos, en primer lugar, en su apariencia manifiesta y, en segundo lugar, cuando la EPL es considerada. De acuerdo con nuestro enfoque metodológico, los citados gráficos muestran la acumulación de vínculos entre los miembros de cada uno de los Gobiernos y, cuando la EPL es tenida en cuenta, la relación previa de los miembros gubernamentales con un conjunto de directivos y dirigentes de grupos de poder pertenecientes a anteriores Gobiernos, al grupo parlamentario, al partido político o a las grandes empresas.

GráFICO 1.

RED DEL GOBIERNO DEL PP (2012)

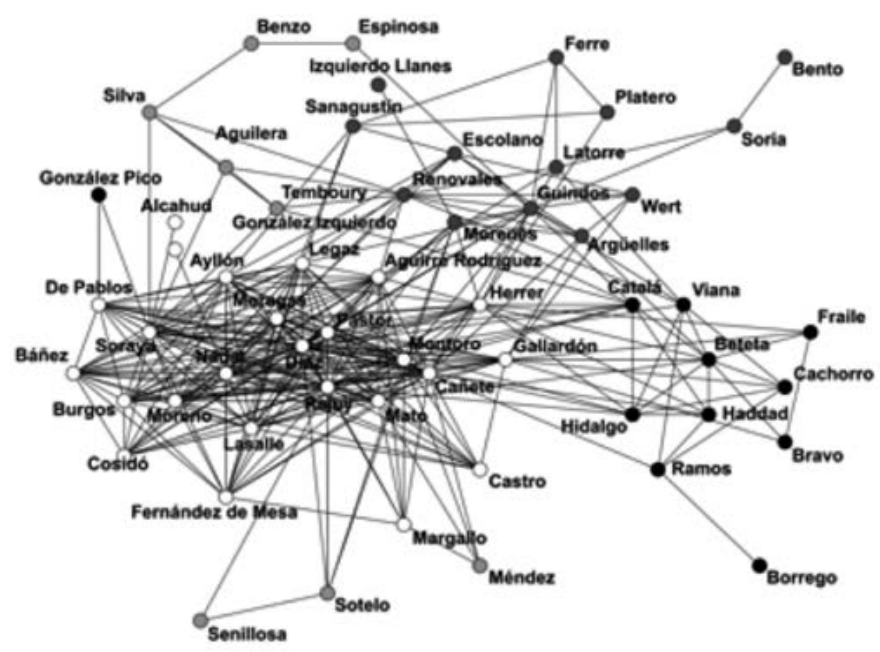

Las distintas escalas de grises identifican facciones con diferentes niveles de cohesión interna. Los nodos aislados $(\mathrm{n}=10)$ no han coincidido con los demás miembros gubernamentales en las dimensiones analizadas. 
Gráfico 2.

Red del Gobierno del PP (2OI2) AL INCluir la EPL

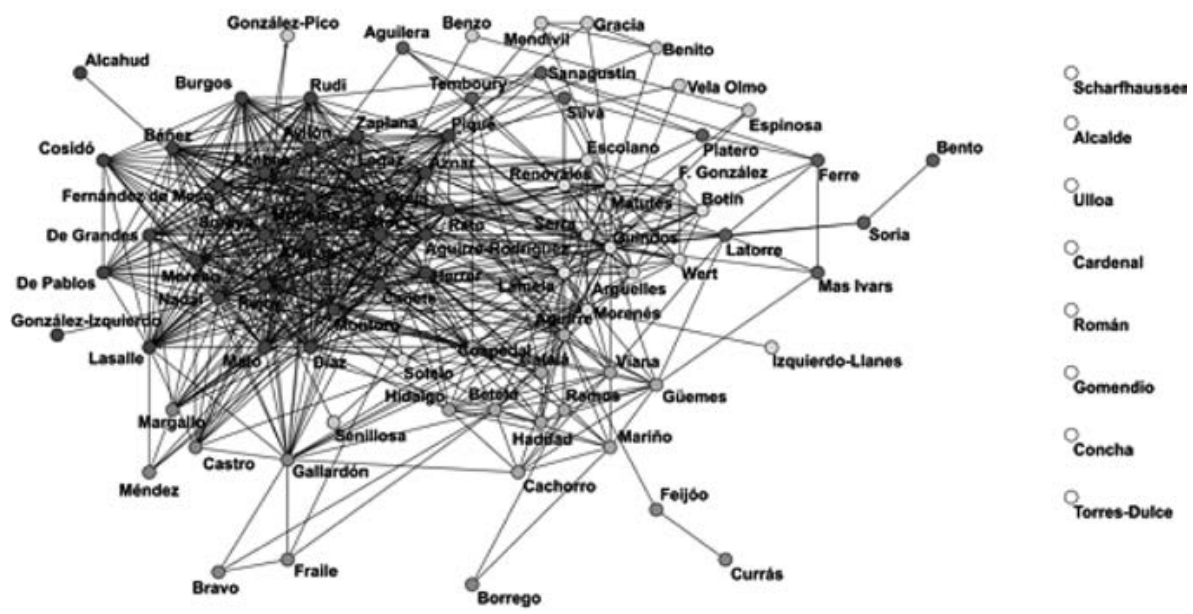

Las distintas escalas de grises definen las distintas facciones sociales con un nivel superior de cohesión interna. Los nodos aislados $(\mathrm{n}=8)$ no coincidieron con ninguno de los otros miembros gubernamentales en las instituciones propuestas.

Fuente: elaboración propia.

GrÁfico 3.

Red del Gobierno del PSOE (2004)
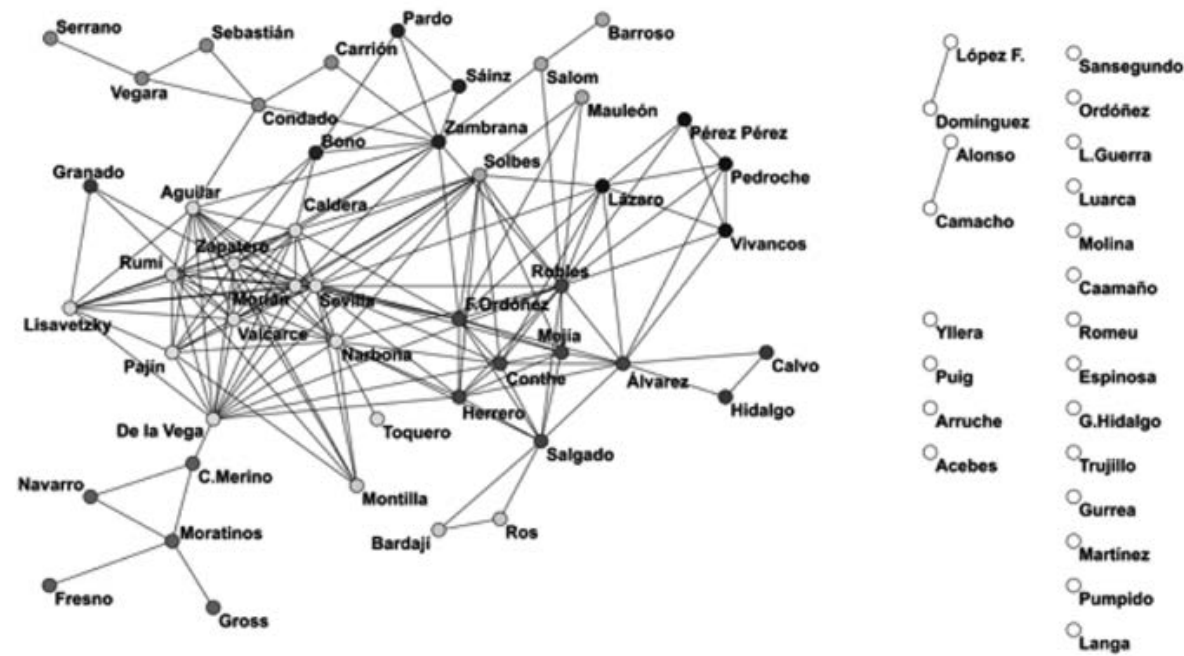

Las distintas escalas de grises definen las distintas facciones sociales con un nivel superior de cohesión interna. Los nodos aislados ( $\mathrm{n}=22$ ) no coincidieron con ninguno de los otros miembros gubernamentales en las instituciones propuestas.

Fuente: elaboración propia. 
GrÁFICO 4.

Red del Gobierno del PSOE (2004) CON EPL

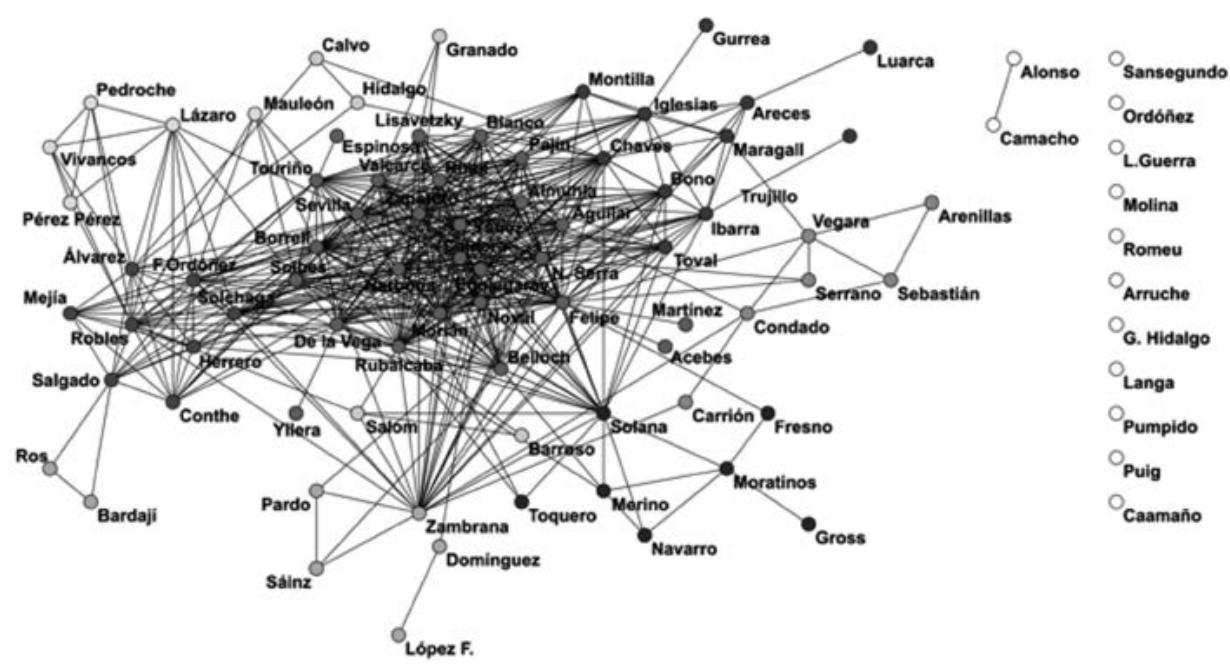

Las distintas escalas de grises identifican facciones con diferentes niveles de cohesión interna. Los nodos aislados $(\mathrm{n}=11)$ no han coincidido con los demás miembros gubernamentales en las dimensiones analizadas.

Fuente: elaboración propia.

Estos gráficos muestran lo que podemos denominar el «grado cero de la formación de un Gobierno", es decir, la estructura relacional de un Ejecutivo al comienzo de una legislatura a partir de los vínculos establecidos en una serie de dimensiones institucionales que consideramos fundamentales para el estudio de las relaciones de poder. Los gráficos mostrados nos ofrecen un primer dato que considerar: la cantidad de relaciones acumuladas en el pasado por los miembros del Gobierno del año 2004 (PSOE) es claramente inferior a la del 2012 (PP), si no se considera la EPL (gráficos 1 y 3). Permite explicar esta diferencia el hecho de que el órgano ejecutivo del PSOE, la Comisión Ejecutiva Federal, fue completamente renovado en el año 2000 (después de dos derrotas electorales consecutivas, la última de ellas, por mayoría absoluta), cuando José Luis Rodríguez Zapatero ganó las elecciones primarias de su partido. El hecho de que muchos de los principales altos cargos del Gobierno del 2004 pertenezcan a la generación de Rodríguez Zapatero y no hayan acumulado muchas relaciones entre sí origina una red de un nivel inferior de cohesión interna que se manifiesta visualmente en un número menor de lazos sociales entre los actores de la red que, además, cuenta con un número superior de nodos o actores aislados con respecto a la del PP. Por el contrario, el órgano ejecutivo del PP, el Comité Ejecutivo Nacional, no se vio sustancialmente alterado de manera previa a la llegada del partido al poder político en el 2012, probablemente debido al hecho de que las derrotas del PP en el 2004 y en el 2008 fueron mucho más ajustadas que la sufrida por el PSOE en el año 2000, con la inmediata dimisión posterior del secretario general Joaquín Almunia y la convocatoria de las elecciones primarias del 2000. 
Estos gráficos muestran también diversas áreas relacionales en escalas de grises. Dichas áreas registran mayores grados de cohesión interna y han sido obtenidas con la aplicación del algoritmo del programa Pajek Factions (De Nooy et al., 2005), que segrega y destaca los subgrupos de nodos que registran un número mayor de relaciones internas con respecto a los vínculos establecidos con el resto de los actores de la red. El conocimiento de la procedencia de los actores miembros de los Gobiernos permite comprobar que la delimitación de facciones se corresponde con el origen institucional de los miembros de los mencionados subgrupos, de tal modo que los componentes gubernamentales que se relacionaron anteriormente y de manera constante en un determinado ministerio aparecerán formando parte de la misma facción relacional en el Gobierno observado.

Con el objeto de analizar cómo afecta la introducción de la EPL a la estructura de los dos Gobiernos y qué diferencias existen entre ellos, se resumen a continuación los resultados de los análisis de facciones para cada caso.

\section{Diferentes maneras de ostentar el poder}

a. Facciones políticas

En ambos casos, se distingue una facción política que, en el PP (ocupando una posición central), está formada por actores de procedencia partidista, parlamentaria y gubernamental y, en el PSOE (en posición central, a la izquierda), por actores exclusivamente partidistas y parlamentarios, debido a su falta de experiencia en Ejecutivos previos. Estas facciones centrales y políticas se ven notablemente expandidas en ambos casos cuando la EPL es considerada. El aumento se debe, en los dos Ejecutivos, a la entrada de un grupo de exministros que participaron en los Gobiernos anteriores del mismo signo ideológico, y que pasan a ocupar posiciones de mediación entre los miembros de la facción política y los de la facción tecnocrática y empresarial. En el caso del Gobierno del PP, estos exministros miembros de la EPL ocupan actualmente posiciones relevantes en grandes empresas privadas, la mayoría de ellas, pertenecientes al índice bursátil español IBEX 35. A diferencia del gobierno del PP, los miembros políticos (también exministros) de la EPL correspondiente al PSOE apenas ocupaban en 2004 puestos en los consejos y equipos directivos de las empresas privadas, ya que habían sido principalmente reclutados por instituciones europeas o globales, tales como la Unión Europea, el Banco Mundial o la OTAN, entre otras. Su reclutamiento por parte de las empresas privadas se produce después del 2004, cuando muchos de tales cargos del PSOE empezaron a participar en los órganos directivos de empresas privadas.

\section{b. Facciones tecnocráticas}

El gráfico 1, referente al caso del PP, muestra varias facciones de individuos que participaron en anteriores Gobiernos y que, después de abandonar el cargo gubernamental, 
trabajaron en la dirección de diversas empresas privadas. La facción más relevante entre estas es la que rodea a la facción política central; sus miembros, mayoritariamente abogados del Estado y técnicos comerciales y economistas del Estado, están relacionados con importantes bancos españoles. La importancia de los perfiles burocráticos de élite en las empresas privadas es un rasgo que ha sido reiteradamente estudiado en las élites españolas (Baena, 1999), además de las élites de otros países como Francia (Comet y Pizarro, 2011). Otra de las facciones tecnocráticas del Gobierno del PP, todavía sin tener en cuenta la EPL, es decir, la mostrada en el gráfico 1, corresponde a actores que han trabajado previamente para un Gobierno del mismo signo político y que posteriormente han adquirido posiciones directivas en empresas constructoras. Una vez introducida la EPL en la estructura relacional del PP, según puede comprobarse en el gráfico 2, esta pasa a incluir a los presidentes de los dos bancos españoles más importantes (Santander y BBVA), por entonces, Emilio Botín y Francisco González, algo que permite constatar que la influencia financiera se incrementa después de la consideración de la EPL. En el caso del PSOE, son pocos los actores que poseen experiencia en grandes empresas, normalmente altos burócratas que trabajaron en anteriores gobiernos del PSOE. La inclusión de la EPL apenas modifica la estructura de esta facción, ya que la mayoría de los integrantes de la EPL socialista quedan integrados en la facción política central, ya analizada.

\section{c. Facciones regionales}

En el caso del PP, se distingue una relevante facción regional (en la parte superior derecha del componente principal), formada principalmente por dirigentes de la Comunidad de Madrid, que es una de las regiones más pobladas en España y, además, una de las comunidades autónomas en las que el PP obtuvo mejores resultados en las elecciones regionales del 2011. Esta facción, además, ve incrementado su tamaño con la consideración de la EPL, lo que muestra la clara influencia potencial en el Gobierno estatal. Este tipo de poder regional se ha destacado como una gran fuente de influencia en los Gobiernos descentralizados de Europa (Botella et al., 2011), entre los cuales España constituye un ejemplo notable. Por su parte, las facciones regionales más significativas en la red del PSOE están formadas por actores que proceden del Gobierno autonómico de Andalucía y de Castilla la Mancha. Estas comunidades autónomas estaban siendo gobernadas por el PSOE mediante mayoría absoluta en el momento de la constitución del Gobierno de 2004. La introducción de la EPL ha hecho aparecer en la red gubernamental a algunos presidentes regionales que estaban en el poder autonómico en el 2004 y algunos de cuyos subordinados políticos fueron reclutados por el Gobierno de 2004. Los Gobiernos, tanto del PP como del PSOE, reclutan a importantes dirigentes de las comunidades autónomas donde estos atesoran una clara superioridad electoral. Así, el Gobierno del PP parece prestar especial atención a la Comunidad y al Ayuntamiento de Madrid, mientras que el PSOE se centra en algunas regiones tradicionalmente socialistas, como las de Andalucía, Castilla la Mancha y Extremadura, entre 
otras. La inclusión de las EPL refleja que la formación de un Gobierno no solo implica fuertes conexiones con empresas privadas, como se ha visto, sino también claras relaciones con otros grupos de poder, como los autonómicos. Países administrativamente descentralizados como España consideran a determinados representantes regionales como importantes mediadores en la relación a establecer entre los diversos organismos públicos territoriales que son gobernados por el mismo partido, de modo que esta perspectiva permite asimismo estudiar cómo distintos grupos de poder como el Estado y las autonomías articulan sus relaciones a través de la formación de un Ejecutivo nacional.

\section{Dos diferentes «cadenas de oportunidades»}

Como ya se ha observado, muchos exministros no volvieron al Gobierno en ambos casos. Este hecho ha generado una serie de vacantes en las estructuras gubernamentales analizadas. En el caso de Gobierno del PP, dichas vacantes han sido ocupadas por exsecretarios de Estado que trabajaron, precisamente, con los exministros no reclutados. Por su parte, en el caso del PSOE, los exministros también dejaron importantes vacantes una vez retirados de la política activa. La mayoría de estas vacantes fueron ocupadas en el 2004 por el nuevo equipo político de José Luis Rodríguez Zapatero, si bien existe una mayor ruptura entre las dos generaciones de dirigentes socialistas que en el caso del PP. Como White afirma en su libro Chain of opportunities, en ambos casos se comprueba que tanto las carreras profesionales como las políticas son difícilmente considerables como independientes del resto de las decisiones y de las trayectorias (White, 1970). La EPL permite explicar la trayectoria de movilidad ascendente de algunos de los miembros de los Gobiernos, mostrando dinámicas propias de promoción de los altos cargos. Además, el mecanismo de creación y ocupación de vacantes revelado constituye una versión particular de la circulación y la renovación de las élites. De esta forma, los nuevos Gobiernos están compuestos por una parte, por algunos ministros de períodos anteriores que repiten en el cargo o en el Ejecutivo y, por otra, por exfuncionarios de alto rango promovidos gracias a su experiencia y a los contactos adquiridos en Gobiernos previos.

Este proceso de circulación de élites puede calificarse como «cerrado» o endogámico, ya que la mayoría de los reclutamientos se basan en vínculos forjados en instituciones en fechas previas. Queda confirmada la hipótesis tres, ya que el proceso de circulación y de renovación de élites se produce a partir de la mediación potencial de los exministros y de otros dirigentes de grupos de poder de la sociedad que trabajaron conjuntamente con muchos de los nuevos incorporados.

\section{La dimensión relacional del poder}

Los análisis anteriores han mostrado cómo las principales facciones de los Gobiernos quedan alteradas con la introducción de las EPL, tal y como se expone a continuación. Dichos análisis han mostrado cómo la introducción de las EPL afecta a las 
distintas facciones de los Gobiernos: por una parte, el gobierno del PP ve notablemente incrementado el tamaño de su facción política con la entrada de numerosos exministros que mantienen fuertes conexiones con empresas privadas; la facción regional se incrementa con algunos dirigentes autonómicos, mientras que la facción empresarial no se ve prácticamente alterada. Por otra, el Gobierno del PSOE ve también aumentado el tamaño de su facción política con la entrada de los ministros de los Gobiernos del expresidente Felipe González, así como con el paso a dicha facción política de miembros del subgrupo tecnocrático, que se encuentran fuertemente conectados con dichos exministros; además, las distintas facciones regionales se vinculan entre sí con la incorporación de altos cargos regionales.

Para continuar con las modificaciones de las redes, en esta sección se estudia si la EPL afecta también a los índices de cohesión interna de las estructuras gubernamentales (tablas 1 y 3 ) y, por lo tanto, si una de sus principales características estructurales queda modificada.

TABLA 1.

INDICES DE COHESIÓN DEL GOBIERNO DEL PP (2OI2), CON Y SIN LA EPL

\begin{tabular}{lcccccc}
\hline & Densidad & Nodos & N. Lazos & Grado medio & Diámetro & Conectividad \\
\hline Red original & 0,14 & 70 & 678 & 9,68 & 5 & 0,66 \\
\hline Red con EPL & 0,17 & 90 & 1362 & 15,13 & 5 & 0,83 \\
\hline
\end{tabular}

Fuente: elaboración propia.

TABLA 3.

ÍNDICES DE COHESIÓN DEL GOBIERNO DEL PSOE (2004), CON Y SIN LA EPL

\begin{tabular}{lcccccc}
\hline & Densidad & Nodos & N. Lazos & Grado medio & Diámetro & Conectividad \\
\hline Red original & 0,07 & 69 & 344 & 4,98 & 7 & 0,46 \\
\hline Red con EPL & 0,13 & 89 & 1038 & 11,66 & 5 & 0,72 \\
\hline
\end{tabular}

Fuente: elaboración propia.

En el marco del análisis de la cohesión de una red social, y según el ARS, cabe considerar los siguientes componentes analíticos, que contribuyen a explicar el grado de cohesión interna:

1. Se define como «densidad» el número total de vínculos en relación con el número de lazos potencialmente existentes (Wasserman y Faust, 1994).

2. Por su parte, el "grado medio" hace referencia al promedio de lazos por actor, de tal manera que el «diámetro» mide la mayor distancia geodésica existente en la red (es decir, la distancia más corta entre cada nodo).

3. Finalmente, la «conectividad» consiste en la proporción de nodos de la red que está conectada al componente principal o de mayor tamaño (Borgatti et al., 2002). 
Estos tres índices de cohesión reflejan que cuando se tiene en cuenta la EPL, la cohesión de ambas redes se ve incrementada. De esta forma, en el caso de Gobierno del PP, la densidad es un $21,4 \%$ superior, el grado medio aumenta en un $56,3 \%$, y el número total de vínculos de la red se ve casi duplicado. Por parte del PSOE, la introducción de la EPL prácticamente duplica la densidad, el grado medio se multiplica por algo más del doble, mientras que el número total de vínculos casi se multiplica por tres. La densidad de la red ampliada del PP $(0,17)$, sigue siendo superior a la del PSOE $(0,14)$, pero las cifras de cohesión se han aproximado notablemente. Precisamente, los datos de cohesión del PSOE revelan que la EPL ejerce sobre el Gobierno del 2004 un grado de influencia mayor del esperado. Este incremento de cohesión era previsible, pues los criterios de construcción de las respectivas EPL exigen de sus miembros haber nombrado a uno o varios de los componentes del Gobierno, lo que trae aparejado lazos con estos. No obstante, también se debe tener en cuenta que índices como la densidad o el grado medio no solo tienen en cuenta el número de conexiones y de lazos sociales incorporados, sino que dividen dicho incremento por el número total de nodos o de actores de la red, que también aumenta. En este sentido, merece la pena destacar el notable incremento en las medidas de cohesión, así como el hecho de que el PSOE vea incrementada su cohesión en mucho mayor grado que el PP, lo que refleja su vinculación potencial a los exgobernantes.

A pesar de la mencionada renovación política en el año 2000, la falta de experiencia gubernamental del equipo liderado por José Luis Rodríguez Zapatero implicó recurrir a los cuerpos técnicos y burocráticos procedentes de anteriores Ejecutivos socialistas. Los exministros para los que dichos técnicos trabajaron pertenecen a la EPL, por lo que vendrían a influir y mediar, con carácter potencial, entre la facción política y la burocrática-técnica del Gobierno del 2004.

En ambos casos se observa que las estructuras de gobierno ampliado son más cohesivas que las originales. Las EPL han permitido describir una comunidad de intereses que tiene su origen en experiencias gubernamentales pasadas. Estas comunidades exhiben dos características destacables:

1. Por una parte, exceden la definición tradicional de Gobierno, generalmente sujeta al conjunto de las posiciones oficiales que componen esta agencia pública.

2. Por otra parte, implica la conexión de dicha agencia pública con un amplio conjunto de grupos de poder, tales como las empresas privadas.

\section{Los actores con mayor influencia potencial}

Ya se ha comprobado que el nivel de cohesión de las redes aumenta con la introducción de las EPL. Un análisis del centro y de la periferia reticular proporciona, además, información relevante acerca de la posición adquirida por los distintos miembros de la EPL en la red ampliada. Al aplicar el algoritmo «núcleo-periferia» del programa Ucinet (Borgatti et al., 2002), por medio del cual se genera el conjunto de actores 
entre los cuales la densidad está maximizada, se obtiene un grupo de ministros y secretarios de Estado que permanecen en el núcleo en ambos escenarios, y que conviven, simultáneamente, con un determinado grupo de exministros. Estas áreas especialmente cohesivas pueden actuar como espacios políticos virtuales que superan los límites formales de un Gobierno, deviniendo en sedes potenciales para la adopción de decisiones relevantes en el transcurso de un mandato político considerado. Esta es una de las mejores virtualidades de la herramienta metodológica construida, en la medida que señala la existencia de áreas relacionales que escapan a la observación si nos limitamos a los datos oficiales.

Con el objeto de obtener información adicional sobre los actores más centrales de cada red, se calcula su nivel de centralidad, entendida esta como el número de relaciones que cada actor o nodo tiene en una determinada estructura relacional (Scott, 2000). La centralidad de grado consiste en el número total de vínculos de cada actor, de tal suerte que cuantas más relaciones tiene un nodo, más conexiones y mejor acceso a la información poseerá (Freeman, 1979). Además, si se mide la centralidad de grado ponderada, no solo se calcula el número de lazos de cada actor, sino también el valor de cada lazo (es decir, el número de años de relación). Por último, la centralidad de intermediación detecta los nodos cuya posición social posee el mayor número de distancias geodésicas (es decir, las distancias más cortas entre los actores de una red en particular). Esta característica posicional otorga la capacidad de mediar entre las diferentes facciones dentro de una red social (Freeman, 1979). Las tablas 2 y 4 muestran, a continuación, los diez primeros actores para cada medida de centralidad.

TABLA 2.

Centralidades de Grado, de grado ponderada y de intermediación de los miembros GUBERNAMENTALES AL INCLUIR LA EPL (LOS MIEMBROS DE LA EPL APARECEN EN NEGRITA)

\begin{tabular}{lclcll}
\hline \multicolumn{1}{c}{ Nombre } & C. Grado & \multicolumn{1}{c}{ Name } & $\begin{array}{c}\text { C. Grado } \\
\text { ponderada }\end{array}$ & \multicolumn{1}{c}{ Nombre } & C. Intermediación \\
\hline 1. R. Rato & 37 & 1. M. Rajoy & 594 & 1. L. Guindos & 810,26 \\
\hline 2. C. Montoro & 36 & 2. J. Arenas & 446 & 2. E. Aguirre & 502,73 \\
\hline 3. M. Cańete & 36 & 3. C. Montoro & 429 & 3. S. Santamaría & 473,08 \\
\hline 4. M. Rajoy & 35 & 4. J. Oreja & 412 & 4. E. Herrer & 399,02 \\
\hline 5. A. Pastor & 35 & 5. R. Rato & 395 & 5. J. Piqué & 345,17 \\
\hline 6. J. Díaz & 35 & 6. A. Mato & 374 & 6. M. Cańete & 309,72 \\
\hline 7. J. Oreja & 32 & 7. A. Pastor & 360 & 7. M. Rajoy & 309,65 \\
\hline 8. S. Santamaría & 31 & 8. J. Aznar & 358 & 8. M. Lamela & 290,64 \\
\hline 9. E. Herrer & 31 & 9. J. Moreno & 344 & 9. R. Rato & 265,10 \\
\hline 10. L. Guindos & 31 & 10. L. Rudi & 333 & 10. A. Matutes & 244,89 \\
\hline
\end{tabular}

Fuente: elaboración propia. 
TABLA 4 .

Centralidades de GRAdo, de GRAdo PONDERADA Y DE INTERMEdiación DE LOS Miembros GUbernAMENTALES AL INCLUiR LA EPL (LOS MIEMbros DE LA EPL APARECEN EN NEGRITA)

\begin{tabular}{lclclc}
\hline \multicolumn{1}{c}{ Nombre } & C. Grado & \multicolumn{1}{c}{ Nombre } & $\begin{array}{c}\text { C. Grado } \\
\text { ponderada }\end{array}$ & \multicolumn{1}{c}{ Nombre } & C. \\
\hline 1. F. González & 36 & 1. F. González & 521 & 1. F. González & 844,37 \\
\hline 2. J. Borrell & 35 & 2. J. Almunia & 453 & 2. J. Solana & 504,15 \\
\hline 3. J. Zapatero & 32 & 3. J. Borrell & 416 & 3. M. Chaves & 471,23 \\
\hline 4. A. Rubalcaba & 32 & 4. N. Serra & 401 & 4. J. Zambrana & 452,48 \\
\hline 5. M. Chaves & 32 & 5. L. Noval & 380 & 5. E. Robles & 370,58 \\
\hline 6. J. Sevilla & 30 & 6. J. Zapatero & 372 & 6. L. Noval & 324,06 \\
\hline 7. J. Caldera & 30 & 7. M. Chaves & 364 & 7. E. Salgado & 296,18 \\
\hline 8. L. Noval & 29 & 8. L. Yánez & 360 & 8. J. Borrell & 270,75 \\
\hline 9. N. Serra & 29 & 9. J. Solana & 358 & 9. A. Rubalcaba & 268,79 \\
\hline 10. C. Narbona & 29 & 10. J. Caldera & 332 & 10. M. Vega & 255,64 \\
\hline
\end{tabular}

Fuente: elaboración propia.

En el caso del PP, un gran número de ministros del 2012 siguen siendo centrales al considerar la EPL. El hecho de que la nueva élite gubernamental esté fuertemente vinculada al pasado ha conducido a un suave proceso de circulación, lo que incrementa el carácter cerrado y endogámico de esta. El dato más destacado lo indica la centralidad de intermediación, en la que destacan varios miembros de la EPL, que se sitúan mayoritariamente entre los perfiles más políticos del Gobierno y los más tecnocráticos. Además de la posesión de esta posición mediadora, la mayoría de estos son exministros que forman parte de los órganos directivos de grandes empresas que pueden constituir un puente entre la estructura gubernamental y el entramado empresarial. En el caso del PSOE, los actores más centrales provienen de la EPL, especialmente el expresidente Felipe González (1982-1996). Las posiciones de mayor centralidad de intermediación son ocupadas, bien por miembros de la EPL, bien por altos burócratas reclutados en el 2004 en contacto con dicha EPL. Dichos burócratas trabajaron con miembros de la EPL en Gobiernos anteriores y median entre la EPL y el núcleo del ejecutivo del 2004. Hay que destacar que el presidente del Gobierno en el año 2004, José Luis Rodríguez Zapatero, ocupa la posición vigésima en esta centralidad; esto indica un déficit de contactos con la facción tecnocrática, pero no representa suficiente evidencia empírica como para afirmar que el entonces presidente no contara con la suficiente capacidad para mediar entre las distintas facciones de la élite. No obstante, los líderes de etapas previas constituyen, todavía en el 2004, un conjunto de actores dotados de una importante influencia política en términos potenciales, a pesar del discurso de renovación política esgrimido por el entonces candidato electoral. 


\section{CONCLUSIONES}

Los análisis aquí expuestos muestran la importancia diferencial de la EPL en dos Gobiernos constituidos en el marco del sistema político español contemporáneo. Las redes gubernamentales ampliadas reflejan un mayor nivel de cohesión interna, de acuerdo con lo enunciado en la hipótesis 1 . Aunque hay claras diferencias entre los dos Gobiernos, los niveles de cohesión interna se aproximan mucho cuando se tiene en cuenta la EPL. Además, la introducción de la EPL supone un incremento de los grupos de poder a los que los Ejecutivos quedan conectados. En el caso del PP, se comprueba que al considerar la EPL, la élite gubernamental del 2012 adquiere un mayor nivel de conexión potencial a grandes empresas y bancos pertenecientes al IBEX 35 en un contexto de turbulencias financieras y de elevada deuda pública, en el que los contactos prioritarios con los intermediarios financieros constituyen un importante activo relacional. Sin embargo, la estructura ampliada del Gobierno del PSOE muestra menos vínculos con empresas privadas, pero importantes relaciones con Gobiernos regionales en los que el PSOE se encontraba gobernando en el 2004. Estas diferentes preferencias pueden deberse a las distintas tendencias ideológicas y, por lo tanto, a distintos grupos de referencia (Merton, 1968), pero también a las variadas circunstancias económicas preponderantes. No obstante, cabría esperar que estas diferencias ideológicas se vean matizadas dada la reciente y progresiva entrada de numerosos miembros de la EPL socialista en los órganos directivos de las grandes empresas.

Los recursos de poder aumentan en ambos casos tras la introducción de las EPL, conforme se formuló por medio de la hipótesis 2 , no solo desde una perspectiva individual, sino también a la luz de una aproximación estructural. Así:

1. En primer lugar, muchos de los miembros reclutados por el gobierno del 2004 ven aumentada su centralidad de grado al ser considerada la EPL, lo que les otorga un mayor grado de poder relacional.

2. En segundo lugar, la cohesión y los vínculos a los grupos de poder se ven significativamente incrementados.

Siguiendo los análisis de Moore (1979) y Higley et al. (1991), y considerando que la cohesión interna y los vínculos a los centros de adopción de decisiones políticas y empresariales constituyen características esenciales de una estructura social de poder, podemos concluir que la EPL contribuye a mostrar un mapa más claro de los recursos de poder que son movilizados y que concurren durante la formación de un Gobierno, proceso, a su vez, incardinado dentro del fenómeno continuo de circulación de las élites.

En relación con el proceso de circulación de élites que tiene lugar (hipótesis 3), podemos referirnos a un proceso de circulación bloqueada o endogámica, que conecta con las reflexiones de Mills (1956). En este sentido, los miembros del Gobierno son en su mayoría reclutados en la medida en que establecieron vínculos en el pasado con los principales ministros en el año 2012, o bien con anteriores altos cargos 
gubernamentales que no fueron reclutados y que, por tanto, se sitúan en otras instituciones de la estructura social. La confianza política y el capital relacional son cruciales para el reclutamiento como ministro, reflejándose un esquema de «cadena de oportunidades» (White, 1970), en el que ciertas características del individuo son relevantes, si bien en combinación con redes profesionales y políticas. Estos procesos de circulación bloqueada indican que la reproducción social de los Gobiernos democráticos depende también del reclutamiento de líderes empresariales con numerosas conexiones en el mundo de las corporaciones privadas. Dicha estructura cerrada de Gobierno corre el riesgo de separarse progresivamente de los intereses de los electores, incidiendo, en su caso, en una eventual crisis de representación democrática.

El enfoque relacional aquí expuesto permite abordar, de una manera alternativa, el análisis de las relaciones entre las agencias públicas y las privadas. Así, en lugar de estudiar el entramado formal e institucional del Estado y las empresas privadas como dos conjuntos separados y, en ocasiones enfrentados, se analiza la estructura de poder como una compleja red social que integra diversas posiciones sociales de influencia permeables entre sí. De esta manera, se destacan áreas relacionales suficientemente cohesivas para constituir sedes de adopción de decisiones relevantes.

\section{AGRADECIMIENTOS}

Los autores desean agradecer a las personas que han evaluado el artículo sus indicaciones y sugerencias, lo que ha redundado en una indudable mejora, tanto en términos formales como sustantivos, del manuscrito original. Agradecen, igualmente, a Delio Lucena Piquero su aportación y ayuda en lo relativo a la elaboración y presentación de los gráficos.

\section{Referencias}

Bachrach Peter y Morton S. Baratz. 1962. "Two faces of power», American Political Science Review, 56: 947-952. Disponible en: https://doi.org/10.2307/1952796.

Baena, Mariano. 1993. Curso de Ciencia de la Administración. Curso de la ciencia de gerencia. Madrid: Tecnos.

Baena, Mariano. 1999. Élites y conjuntos de poder en España (1939-1992). Un estudio cuantitativo sobre Parlamento, Gobierno y Administración y gran empresa. Madrid: Tecnos.

Baena, Mariano y Narciso Pizarro. 1985. «The structure of the Spanish political elite (1939-1975)», en Gwen Moore, ed., Studies on the structure of national elite groups. Researchs in Politics and Society, volume 1. Nueva York: JAI Press.

Bobbio, Norberto. 2013. Democracia y secreto. Ciudad de México: FCE, 2013.

Borchert, Jens. 2003. «Professional Politicians: Towards a Comparative Perspective», en Jens Borcherty Jürgen Zeiss (eds.), The Political Class in Advanced Democracies. 
Oxford: Oxford University Press. Disponible en: https://doi.org/10.1093/01992 60362.003 .0001 .

Borgatti, Steve, Martin G. Everett y Linton C. Freeman. 2002. Ucinet for Windows: Software for Social Network Analysis. Harvard: Analytic Technologies.

Botella, Joan, Joan Rodríguez, Óscar Barberá, y Astrid Barrio. 2011. «Las carreras políticas de los jefes de Gobierno regionales en España, Francia y el Reino Unido (1980-2010). Las carreras políticas de los jefes ejecutivos regionales en España, Francia y el Reino Unido (1980-2010)», Revista Española de Investigaciones Sociológicas, 133: 3-20. Disponible en: https://doi.org/10.5477/cis/reis.133.3.

Bottomore, Tom. 1993. Elites and society. Londres: Routledge.

Burnham, James. 1943. The machiavellians. Defenders of freedom. Reprint 1988. Nueva York: Gateway Editions.

Canales, José M. 2015. Estudios sobre democracia, Gobierno y Administración pública contemporánea. Madrid: Universitas.

Castells, Manuel (ed.). 2006. La sociedad red: una visión global. Madrid: Alianza.

Cervera, Juan A. 1984. La red del poder. Madrid: Ediciones Dyrsa.

Comet, Catherine y Narciso Pizarro. 2011. "The cohesion of intercorporate networks in France», Procedia Social and Behavioral Sciences, 10: 52-61. Disponible en: https://doi.org/10.1016/j.sbspro.2011.01.008.

Dahl, Robert. 1960. Who governs? Reprint 2005. New Haven y Londres: Yale University Press.

De Nooy, Wouter, Andrej Mrvar y Vladimir Batagelj. 2005. Exploratory Social Network Analysis with Pajek. Cambridge: Cambridge University Press. Disponible en: https://doi.org/10.1017/CBO9780511806452.

Degenne, Alain y Michel Forsé. 1999. Introducing Social Networks. Londres: Sage.

Disponible en: https://doi.org/10.4135/9781849209373.

Del Campo, María. 2014. «Gobernanza, democracia y políticas públicas», en Gema Pastor (coord.), Teoría y práctica de las politicas públicas. Valencia: Tirant Lo Blanch.

Domhoff, William. 1969. ¿Who rules America? Reprint 2009. Englewood Cliffs, Nueva Jersey: Prentice Hall.

Freeman, Linton. 1979. "Centrality in Social Networks. Conceptual clarification», Social Networks, 1: 215-239. Disponible en: https://doi.org/10.1016/0378-8733 (78)90021-7.

Gil Mendieta, Jorge y Samuel Schmidt. 2005. Estudios sobre la red politica de México. Un estudio realizado en México la red de politicos. Ciudad de México: Universidad Autónoma de México.

Haas, Peter M. 1989. "Do regimes matter? Epistemic communities and the Mediterranean pollution control», International Organization, 43 (3): 377-403. Disponible en: https://doi.org/10.1017/S0020818300032975

Haas, Peter M. 1992. «Introduction: Epistemic communities and international policy coordination», International Organization, 46 (1): 1-35. Disponible en: https:// doi.org/10.1017/S0020818300001442. 
Hanneman, Robert y Mark Riddle. 2005. Introduction to social network methods. Riverside: University of California.

Heinz, John, Edward Laumann, Robert Nelson, y Robert Salisbury. 1993. The hollow core, private interests in national policy making. Cambridge: Harvard University Press.

Hoffmann-Lange, Ursula. 1989. "Positional power and political influence in the Federal Republic of Germany», European Journal of Political Research, 17 (1): 51-76. Disponible en: https://doi.org/10.1111/j.1475-6765.1989.tb00181.x

Highley, John, Ursula Hoffmann-Lange, Charles Kadushin, y Gwen Moore. 1991. «Elite integration in stable democracies: a reconsideration», European Sociological Review, 7 (1): 35-53. Disponible en: https://doi.org/10.1093/oxfordjournals.esr. a036576.

Kadushin, Charles. 2012. Understanding social networks: theories, concepts and findings. Oxford: Oxford University Press.

Kenis, Patrick y Volker Schneider. 1991. «Policy Networks and Policy Analysis: Scrutinizing a New Analytical Toolbox», en Bernd Marin y Renate Mayntz (eds.), Policy Networks: Empirical Evidence and Theoretical Considerations. Frankfurt: Westview Press.

Knoke, David. 2001. Changing Organizations: Business Networks in the New Political Economy. Boulder: Westview Press.

Kooiman, Jan. 2005. «Gobernar en gobernanza», en Agustí Cerrillo (ed.), La gobernanza hoy: 10 textos de referencia. Madrid: INAP.

Laumann, Edward, Peter Marsden y Joseph Galaskiewicz. 1977. "Community Influence Structures: Replication and Extension of a Network Approach», American Journal of Sociology, 31:169 78.

Laumann, Edward y Franz Pappi. 1976. Networks of collective action: a perspective on community influence systems. Nueva York: Academic Press.

Laver, Michael y Kenneth Shepsle. 1996. Making and breaking governments. Cabinet and legislatures in parliamentary democracies. Londres: Cambridge University Press. Disponible en: https://doi.org/10.1017/CBO9780511625671.

Luengo, Jorge. 2016. "Las élites liberales: una sociedad conyugal», Historia Social, 86: 91-108.

Lusher, Dean, Johan Koskinen y Garry Robins (eds.). 2013. Exponential Random Graph Models for Social Networks. Cambridge: Cambridge University Press.

Meisel, James. 1958. The myth of the ruling class: Gaetano Mosca and "The Elite». Ann Arbor: The University of Michigan Press.

Merton, Robert. 1968. Social theory and social structure. Nueva York: The Free Press.

Michels, Robert. 1915. Political parties. A sociological study of the oligarchical tendencies of modern democracy. Reprinted 1961. Nueva York: The Free Press.

Miliband, Ralph. 1969. The state in capitalist society. Reprint 2009. Londres: Merlin Press. Mills, Charles W. 1956. The power elite. Reprint in 2000. New York: Oxford University Press.

Moore, Gwen. 1979. «The structure of a National Elite Network», American Sociological Review, 44 (5): 673-692. Disponible en: https://doi.org/10.2307/2094520. 
Morgan, Glenn, Paul Hirsch and Sigrid Quack (eds.). 2015. Elites on trial. Bingley: Emerald Group. Disponible en: https://doi.org/10.1108/S0733-558X201543.

Mosca, Gaetano. 1939. The ruling class (Elementi di Scienza Politica). Reprint 1979. Nueva York y Londres: McGraw-Hill.

Musella, Fortunato. 2015. «Presidents in business. Career and destiny of democratic leaders», European Political Science Review, 7 (2): 293-313. Disponible en: https:// doi.org/10.1017/S1755773914000149.

Pareto, Vilfredo. 1917. Traité de sociologie générale. Reprint 1968. Ginebra: Droz.

Parrado, Salvador. 1996. Las élites de la administración estatal (1982-1991). Estudio general y pautas de reclutamiento. Sevilla: Instituto Andaluz de Administración Pública.

Prats, Joan. 2005. De la burocracia al management. Del management a la gobernanza. Las transformaciones de las Administraciones Públicas de nuestro tiempo. Madrid: INAP.

Polsby, Nelson. 1980. Community power and political sociology. New Haven: Yale University Press.

Putnam, Robert. 1976. The comparative study of political elites. Englewood Cliffs: Prentice-Hall.

Rhodes, Roderick y David Marsh. 1992. "New directions in the study of policy networks», European Journal of Political Research, 21: 181-205.

Rodríguez Teruel, Juan. 2010. «¿Gobierno parlamentario sin ministros parlamentarios? La influencia de la descentralización en las carreras de la élite ministerial española», Revista Española de Ciencia Politica, 24: 83-105.

Rose, Richard (ed.). 1987. Ministers and ministries. A functional analysis. Oxford: Clarendon Press.

Sánchez de Dios, Manuel. 2006. «Nueva perspectiva de la responsabilidad política. La teoría de la agencia», Revista de las Cortes Generales, 67: 139-181.

Scott, John. 2000. Social Network Analysis: a Handbook. Newbury Park, CA: Sage.

Suleiman, Ezra y Henri Mendras (eds.). 1995. Le recruitment des élites en Europe. París: La Découverte.

Thiébault, Jean L. 1991. "Local and regional politics and Cabinet Membership», en Jean Blondel y Jean L. Thiébault, The Profession of Government Minister in Western Europe. Londres: Mc. Millan. Disponible en: https://doi.org/10.1007/978-1-34911395-8_4.

Tuñón de Lara, Manuel. 1967. La historia y la realidad del poder: el poder y las elites durante el primer tercio del siglo XX en España. Madrid: Edicusa.

Van Apeldoorn, Bastiaan y De Graaff, Nana. 2014. «Corporate elite networks and US post-Cold War grand strategy from Clinton to Obama», European Journal of InternationalRelations, 20(1):29-55.Disponibleen:https://doi.org/10.1177/1354066111 433895.

Visser Jelle y Mark Van Der Meer. 2010. "The Netherlands: Social Pacts in a Concertation Economy», en Sabina Avdagic, Martin Rhodes y Jelle Visser (eds.), Social 
Pacts in Europe: Emergence, Evolution, and Institutionalisation. Oxford: Oxford University Press [forthcoming].

Wasserman, Stanley y Katherine Faust. 1994. Social network analysis. Methods and applications. Cambridge: Cambridge University Press. Disponible en: https://doi. org/10.1017/CBO9780511815478.

Weber, Max. 1922. Economy and society. An outline of interpretive sociology. Reprint 1988. California: California University Press.

White, Harrison. 1970. Chains of opportunity: system models of mobility in organizations. Cambridge: Harvard University Press. Disponible en: https://doi.org/10.4159/ harvard.9780674437203

Presentado para evaluación: 5 de julio de 2017.

Aceptado para publicación: 27 de octubre de 2017.

ANDRÉS VILLENA-OLIVER

villenaoliver@gmail.com

Doctor en Sociología y licenciado en Economía y en Comunicación Audiovisual por la Universidad de Málaga.

\section{BERNABÉ ALDEGUER-CERDÁ, UNIVERSIDAD DE ALICANTE}

bernabe.aldeguer@ua.es

Doctor en Estudios Políticos y Constitucionales por la Universidad de Alicante y licenciado en Ciencias Políticas y de la Administración. En la actualidad es profesor de Ciencia Política y de la Administración en la Universidad de Alicante. 